Man and Nature

L'homme et la nature

\title{
Libertinage et Révolution : Les Romans du Marquis de Sade
}

\section{Marie-France Silver}

Volume 8, 1989

URI : https://id.erudit.org/iderudit/1012604ar

DOI : https://doi.org/10.7202/1012604ar

Aller au sommaire du numéro

Éditeur(s)

Canadian Society for Eighteenth-Century Studies / Société canadienne d'étude du dix-huitième siècle

ISSN

0824-3298 (imprimé)

1927-8810 (numérique)

Découvrir la revue

Citer cet article

Silver, M.-F. (1989). Libertinage et Révolution : Les Romans du Marquis de Sade. Man and Nature / L'homme et la nature, 8, 129-136.

https://doi.org/10.7202/1012604ar

Copyright (C Canadian Society for Eighteenth-Century Studies / Sociéte canadienne d'étude du dix-huitième siècle, 1989
Ce document est protégé par la loi sur le droit d'auteur. L'utilisation des services d'Érudit (y compris la reproduction) est assujettie à sa politique d'utilisation que vous pouvez consulter en ligne.

https://apropos.erudit.org/fr/usagers/politique-dutilisation/ 


\section{Libertinage et Révolution: Les Romans du Marquis de Sade}

$C^{\prime}$ est en tant qu'auteur ${ }^{1}$ et non pour les désordres de sa vie privée que Sade fut arrêté en 1803 et interné à Charenton qui offrait l'avantage du point de vue du pouvoir d'être à la fois asile psychiatrique et prison politique. ${ }^{2} \mathrm{C}^{\prime}$ est là qu'il mourut en 1812 sans avoir jamais obtenu le procès qu'il réclamait.

Je propose d'analyser le libertinage sadien pour essayer de comprendre pourquoi les générations issues de la Révolution ont cherché à ensevelir cette oeuvre dans l'oubli.

Le libertinage sadien se caractérise essentiellement par le double aspect philosophique et pornographique qui ne peuvent être dissociés sans trahir la pensée de l'auteur.

Sade connaissait bien la littérature pornographique du XVIIIe siècle. Il divise ce genre en deux catégories.

La première comprend les ouvrages obscènes pour la plupart anonymes qu'il méprise profondément. Il dénonce:

ces misérables petites brochures faites dans des cafés ou dans des bordels, et qui prouvent à la fois deux vides dans leurs mesquins auteurs, celui de l'esprit et celui de l'estomac. ${ }^{3}$

La seconde catégorie, qui l'intéresse davantage, est une série d'ouvrages relativement connus tels Le Portier des Chartreux, l'Académie des Dames, L'Education de Laure et Thérèse philosophe. Mais il en trouve trop limitée la portée. Le Portier des Chartreux est une production 'plus polissonne [commente-t-il] que libertine. ${ }^{\prime 4}$ L'Académie des Dames est 'un ouvrage fait par un homme timide qui avait l'air de sentir la vérité mais sans la dire. ${ }^{\prime 5}$

La pornographie au service de la vérité, c'est-à-dire en termes sadiens de la philosophie, voilà l'ambition du libertinage de l'auteur. La base philosophique de ce libertinage repose sur une conception épicurienne de la nature qui place l'auteur dans la tradition du libertinage érudit du XVIIe siècle. Rejetant causes premières et causes finales, Sade propose, en effet, dans ses romans une explication mécaniste de l'univers qui remonte à Lucrèce et à Epicure. 
Un seul moteur agit dans l'univers et ce moteur, c'est la nature,

affirme Dolancé dans La Philosophie dans le boudoir. ${ }^{6}$ Le mouvement est inhérent à la matière:

La matière se meut par sa propre énergie, par une suite nécessaire de son hétérogénéité,

est-il expliqué dans La Nouvelle Justine. ${ }^{7} \mathrm{C}^{\prime}$ est dans ce mouvement interne de la matière que l'auteur trouve la clef de l'explication des phénomènes, comme il le dit dans Juliette:

Dieu n'est qu'une modification de la matière perpétuellement en action par son essence, cette action $[\ldots]$, cette énergie de la matière, voilà votre Dieu. ${ }^{8}$

$\mathrm{Du}$ dynamisme de la matière découlent les idées épicuriennes du passage de l'inerte au sensible, de la permanence du tout et de la variation perpétuelle des formes:

A notre mort [déclare un personnage de La Nouvelle Justine], c'est-à-dire à la réunion des éléments qui nous composent à la masse générale, nous passerons un instant dans le creuset de la nature pour en rejaillir sous d'autres formes. ${ }^{9}$

Dans la lignée du libertinage érudit du XVIIe siècle et des épicuriens du XVIIIe, Sade essaie de montrer que l'homme est nature, qu'animalité et intelligence sont les deux modes d'une même réalité physique ou corporelle. Ce qui le différencie cependant de ses prédécesseurs, c'est la conception tragique qu'il offre de la nature:

Plus j'ai cherché à surprendre ses secrets [ecrit-il], plus je l'ai vue uniquement occupée à nuire aux hommes. Suivez-la dans toutes ses opérations, vous ne la trouverez jamais que vorace, destructrice et méchante, jamais qu'inconséquente, contrariante et dévastatrice. ${ }^{11}$

L'univers où se meuvent ses héros est bien l'univers de Lucrèce où les hommes [écrit Sade] ne sont que 'le résultat des lois aveugles de la nature'. ${ }^{12}$ Ceci permet de mieux comprendre la haine des héros sadiens pour cette dernière, cette 'force aveugle et imbécile' dont ils se trouvent, disent-ils, 'le résultat involontaire' ${ }^{13} \mathrm{~A}$ une époque qui avait érigé en culte l'idée d'une nature bienveillante, féconde et souriante héritée des Lumières, Sade, en proclamant le caractère destructeur et amoral de la nature, désacralise cette dernière: 
Des grands et des petits la putain est la mère, Et nous sommes toujours aussi chers à ses yeux, Monstres et scélérats que bons et vertueux

déclare-t-il dans son poème La Vérité. ${ }^{14}$ Il appuie son entreprise de désacralisation sur la science du temps. Empruntant, en effet, des arguments à l'Histoire naturelle de Buffon et au De la nature de Robinet, ${ }^{15}$ Sade s'acharne à prouver que la destruction est nécessaire à la nature et que la mort, le crime, la guerre, le mal assurent l'équilibre biologique de l'univers:

C'est par un mélange absolument égal de ce que nous appelons crime et vertu [explique l'auteur] que [les lois de la nature] nous soutiennent; c'est par des destructions qu'elle renaît; c'est par des crimes qu'elle subsiste; c'est en un mot par la mort qu'elle vit. ${ }^{16}$

Les héros sadiens, en se livrant à un entre-dévorement général, ne font en effet qu'obéir aux pulsions destructrices de la nature. Ainsi, l'idée d'un déterminisme universel, inhérente à l'épicurisme, aboutit chez Sade à la négation de la liberté:

Ce système de liberté est une chimère [assure La Delbène], nous sommes poussés à tout ce que nous faisons par une force plus puissante que nous. ${ }^{17}$

Cette optique exclut toute notion de progrès.

Pour Sade [en effet remarque Jean-Claude Bonnet], l'histoire ne fait que répéter les mêmes mécanismes de l'horreur. [Elle est réduite] à une machine cruelle [qui] engloutit les générations humaines. ${ }^{18}$

La lutte des classes n'est alors qu'un aspect de la destruction universelle de la nature:

Pourquoi [interroge Sade] ne voulons-nous pas [que la nature] ait fait parmi les hommes ce que nous voyons parmi les animaux? toutes les classes ne se dévorent-elles pas mutuellement? ${ }^{19}$

L'on voit ainsi comment l'auteur glisse dangereusement du plan métaphysique au plan social et donc nécessairement à des implications politiques et morales. Il brosse de l'Ancien Régime un des tableaux les plus noirs de la littérature. Dans cette société où l'individu n'est rien, les ordres privilégiés se livrent à une exploitation continue du peuple. Catholicisme et monarchie se soutiennent mutuellement pour mieux 
exercer leur tyrannie. Le voyage de Juliette en Italie ou celui de Brisatesta dans les cours du Nord est un tourisme politique subversif. ${ }^{20}$ Par leur intermédiaire Sade se livre à 'un jeu de massacre des têtes couronnées et un jugement dernier des rois' ${ }^{21}$ et de la papauté. Corruption, débauche, exactions sexuelles, crimes sont présentés comme l'ordinaire des ordres privilégiés et des grands de ce monde. Dans cette optique la pornographie des romans sadiens, comme celle des libelles politico-pornographiques de l'époque ${ }^{22}$ contribue à la démystification des fétiches religieux et politiques de l'Ancien régime. Mais Sade ne s'en tient pas là. Juliette et La Philosophie dans le boudoir dénoncent en effet les mécanismes d'oppression qui continuent à fonctionner après 1789 et même, ce qui devait nécessairement choquer les générations issues de la Révolution, après la chute de la dictature jacobine. Quand dans Juliette Brisatesta décrit le despotisme monarchique, il insiste longuement sur celui de l'opposition qui est animée du même désir de tyranniser. Une note de l'auteur précise que la Révolution française était inspirée par un semblable ressentiment à couleur despotique. ${ }^{23}$ Le pamphlet politique Français encore un effort si vous voulez être républicains montre comment au sein de la république thermidorienne l'inégalité sociale persiste sous le masque $d^{\prime}$ 'une égalité juridique théorique. ${ }^{24}$ L'inégalité des sexes continue à être sanctionnée par le mariage qui opprime bien plus les femmes que les hommes puisqu'il contribue à maintenir les femmes dans un état subalterne et dégradant. La loi de la propriété, sanctionnée par la Déclaration des droits de l'homme et de nouveau en 1795 par la constitution thermidorienne, loin d'être un droit fondamental de l'homme, n'est qu'une usurpation du riche, la caution légale d'une inégalité qui lui est favorable. ${ }^{25}$

De plus, en intégrant délibérément ce pamphlet politique à un ouvrage pornographique, Sade tourne en dérision le discours révolutionnaire. Michel Delon a bien montré dans son article Sade thermidorien que la lecture du pamphlet au milieu des orgies organisées par Mme de St-Ange, renvoyait directement à la situation politique. ${ }^{26} \mathrm{C}^{\prime}$ était en effet l'époque où les thermidoriens se voyaient dénoncés par l'opposition comme des contre-révolutionnaires jouant dans les salons aristocratiques du vocabulaire et du décorum révolutionnaires. ${ }^{27}$ La philosophie dans le boudoir souligne elle aussi l'alliance entre les forces de l'aristocratie représentées par Mme de St-Ange et son frère Dolmancé, et celles de l'argent qu'incarne Eugénie, fille de l"un des plus riches financiers de la capitale.' Sade, dans cet ouvrage, tourne en dérision la révolution bourgeoise. La critique de la politique contemporaine qui colore les romans sadiens n'était pas faite pour disposer favorablement le pouvoir à l'égard de l'auteur. 
Ce que Sade veut montrer dans Juliette, La Philosophie dans le boudoir et dans Français encore un effort si vous voulez être républicains, c'est que la Révolution française n'a pas été une véritable révolution puisque la société reste divisée entre oppresseurs et opprimés, riches et pauvres, sexe fort et sexe faible. La pornographie dans les romans sadiens débouche invariablement sur la politique. Car la main qui frappe, qui fouette, qui torture, qui met à mort est celle qui détient le pouvoir dans la société. En montrant que malgré le changement de régime politique, la Révoltuion ne s'était pas débarrassée des inégalités fondamentales, Sade se livre à une véritable désacralisation de la Révolution française au moment même où cette dernière était justement en train d'être mythifiée. Mais le but de Sade était moins de dénoncer tel ou tel régime politique que de mettre à jour tous les mécanismes qui oppriment l'individu sous divers déguisements.

Est-ce la peine de vivre quand on languit sous le joug du mensonge et de la stupidité?

s'interroge Juliette $^{28}$ qui, à la dernière page d'une 'vie éclaboussée de sperme et de sang, ${ }^{29}$ proclame: 'La philosophie doit tout dire. ${ }^{\prime 30}$

Si l'auteur se fait ainsi l'écho de Kant qui voyait dans le vers d'Horace 'Aie le courage de ton propre entendement' la définition de la philosophie des Lumières, Sade ne fait pas comme Kant la distinction entre l'inaliénable liberté de penser et les devoirs de la vie sociale. ${ }^{31}$ $C^{\prime}$ est là la plus grande différence entre Sade et le rationalisme issu des Lumières. La prise de parole philosophique à l'époque des Lumières et pendant la Révolution s'est faite au nom du bien public. ${ }^{32}$ Celle de Sade se fait constamment au nom de l'individu. C'est pourquoi, au moment où les révolutionnaires s'efforçaient de rédiger un nouveau code législatif, dont Cambacérès rapporte plusieurs projets, Sade dénonce avec acharnement l'emprise de la loi sur l'individu car, remarque-t-il, 'les lois lui prennent infiniment plus qu'elles ne lui donnent. ${ }^{\prime 33}$ Dans Juliette il précise

L'homme n'est-il pas partout l'esclave des lois, et de ce moment, ne le voilà-til pas enchaîné. ${ }^{34}$

Et l'auteur de conclure:

Le régime des lois est ... vicieux; il est donc inférieur à celui de l'anarchie.

L'idée de solidarité qui prend une telle ampleur au cours du siècle ${ }^{36}$ n'est pour lui qu'une chimère: 
Ne naissons-nous pas tous isolés [remarque-t-il], tous ennemis les uns des autres, tous dans un état de guerre perpétuelle et réciproque. ${ }^{37}$

Les rapports de force que Sade a mis à jour dans la vie sexuelle sont le reflet des rapports de force entre les individus

tous les hommes tendent au despotisme [explique-t-il dans Juliette]. C'est le premier désir que nous inspire la nature. J'affirme donc que le premier et le plus vif penchant de l'homme est incontestablement d'enchaîner ses semblables et de les tyranniser de tout son pouvoir. ${ }^{38}$

L'idee d'un sentiment naturel qui inspirerait à l'homme l'amour de la vertu est tournée par Sade en dérision:

L'homme sauvage ne connait que deux besoins [assure-t-il]: celui de foutre et celui de manger; tous les deux viennent de la nature. ${ }^{39}$

La morale de la société n'est donc pas la morale de la nature. La Mettrie avait avant Sade atteint la même conclusion et dénoncé 'les capucinades de la vertu du Système social du baron $\mathrm{d}^{\prime} \mathrm{Holbach},{ }^{40}$ mais il s'était bien gardé de juger néfaste la morale sociale. Pour Sade cette dernière est une imposture, un préjugé qu'il faut à tout prix dénoncer et il le fait avec la même vigueur qu'il avait mise à dénoncer tout système religieux. D'où le rejet que les athées ont fait de Sade et de son oeuvre. A l'aube du XIXe siècle, en effet, l'athéisme s'était promu en une caste vertueuse ${ }^{41}$ qui se faisait un point $d^{\prime}$ honneur de respecter la morale courante.

Je voudrais [écrit en 1805 l'astronome Joseph Jérôme de La Lande dans le supplément au Dictionnaire de athées] pouvoir citer M. de Sade, il a bien assez d'esprit, de raisonnement, d'érudition, mais ses infâmes romans ... le font rejeter d'une secte où l'on ne parle que de vertu. ${ }^{42}$

Bien que le libertinage sadien s'inscrive dans la tradition du libertinage érudit, son caractère particulier lui est conféré par une triple entreprise de désacralisation:

- désacralisation de la nature.

- désacralisation de l'autorité et de la loi.

- désacralisation de la morale.

Ce faisant, il heurtait de front l'athéisme qui était en train de se définir à l'intérieur du cadre de la morale bourgeoise. Il attaquait aussi 
directement les principaux attributs de la mythologie de la Révolution, c'est-à-dire, la croyance au progrès, le culte d'une nature vertueuse, les principes de liberté, d'égalité et de fraternité. Pour les héritiers de la révolution bourgeoise, le libertinage sadien était donc inacceptable.

\section{MARIE-FRANCE SILVER}

Université York

\section{Notes}

1 Marie-France Silver, 'L'Etrange don du Marquis de Sade à la Révolution et à l'Empire: Son théâtre,' Man and Nature, vol. VI (1987), 210-211.

2 Michel Vovelle, Théodore Desorques ou la désorganisation (Paris: Seuil), p. 198.

3 Histoire de Juliette, dans Oeuvres Complètes du marquis de Sade (Paris: Cercle du livre précieux, 1966), 8:442-43.

4 Juliette, 8:442-43.

5 Juliette, 8:442-43.

6 La Philosophie dans le boudoir (Paris: Gallimard, 1976), p. 70.

7 La Nouvelle Justine, dans Oeuvres complètes, 6:208.

8 Juliette, 8:442-43.

9 La Nouvelle Justine, 6:208.

10 La Philosophie dans le boudoir, dans Oeuvres complètes, 6:238.

11 Déjà cité par M. Heine dans Oeuvres complètes, 14:45.

12 Juliette, 9:170-71.

13 Juliette, 9:186-87.

14 La Vérité, dans Oeuvres complètes, 14:45.

15 Cf. Jean Deprun, 'Sade et la philosophie biologique de son temps,' dans Le Marquis de Sade (A. Colin, 1968).

16 Juliette, 8:168.

17 Juliette, 8:25.

18 Jean-Claude Bonnet, 'Sade historien,' dans Sade: écrire la crise (Paris: Belfond, 1983).

19 Juliette, 8:168-9.

$20 \mathrm{Cf}$. à ce sujet l'article de Bonnet.

21 L'expression est empruntée à Bonnet. 
22 Cf. Robert Darnton, Bohème littéraire et Révolution (Paris: Seuil, 1983), pp. 96-102.

23 Ceci a été noté par Bonnet.

24 Cf. l'article de Michel Delon, 'Sade thermidorien' dans Sade: écrire la crise.

25 La Philosophie dans le boudoir, pp. 213-14.

26 Cf. Delon, 'Sade thermidorien.'

27 Delon, 'Sade thermidorien.'

28 Juliette, 8:60.

29 L'expression est de Michel Delon dans son article 'Homo sum . . .,' DixHuitième Siècle, 16 (1984):294.

30 Juliette, 9: Fin.

31 Delon, 'Homo sum . . .

32 Delon, 'Homo sum . . .,' p. 294.

33 Juliette, 8:172.

34 Juliette, 9:516.

35 Juliette, 9:137.

36 Cf. Delon, 'Homo sum . . .'

37 La Philosophie dans le boudoir, p. 170.

38 Juliette, 8:305.

39 Juliette, 9:513.

40 Cité par A. Adam, dans Oeuvres complètes, 14:23.

41 Cf. Heine, 14:42.

42 Cité par Heine, 14:41. 